\title{
Compreensão do significado de família por estudantes universitários baianos
}

\section{Comprehension of the meaning of family by university students from Bahia}

Elaine Pedreira Rabinovich*

Universidade Católica do Salvador - UCSAL, Salvador, Bahia, Brasil

Anamélia Lins e Silva Franco**

Universidade Católica do Salvador - UCSAL, Salvador, Bahia, Brasil

Lúcia Vaz de Campos Moreira***

Universidade Católica do Salvador - UCSAL, Salvador, Bahia, Brasil

\begin{abstract}
RESUMO
Como parte complementar de um estudo quantitativo sobre papéis familiares, 170 universitários da Bahia responderam a um questionário aberto sobre composição, definição e ideal de família. As respostas a estas questões foram organizadas em categorias por análise de conteúdo. Os principais resultados definiram a família como base segura $(59,1 \%)$ e o ideal de família como convivialidade harmônica (52,3\%). Para composição familiar foram estabelecidas 10 categorias (extensa: 45,2\%; nuclear: 22,0\%; extensa + amigos: $10,7 \%$, ampliada: $6,5 \%$; monoparental: 5,9\%; monoparental ampliada: $4,2 \%$ e outras). A principal conclusão foi a de que o retrato da família ideal apontou para um movimento em direção a uma família mais horizontal e menos autoritária do que a família tradicional brasileira.
\end{abstract}

Palavras-chave: Família, horizontalidade, composição familiar, Bahia.

\begin{abstract}
To complement a quantitative study about family roles, 170 university students from Bahia answered an open questionnaire about composition, definition and ideal family. The answers to these questions were organized into categories by content analysis. The main results pointed to a change from family defined as a secure base $(59,1 \%)$ to an ideal conception of family related to harmonic conviviality $(52,3 \%)$. 10 categories were given as to family composition (extended, 45,2\%; nuclear: $22,0 \%$,; extended + friends: 10,7\%; amplified: 6,5\% ; monoparental amplified: 5,9\%; monoparental: $4,2 \%$; others). The main conclusion was that the ideal family portrait pointed to a movement to a more horizontal and less authoritarian family than the traditional Brazilian family.

Keywords: Family, horizontality, family composition, Bahia.
\end{abstract}




\section{I ntrodução}

Esta pesquisa foi baseada no estudo Families across cultures. A 30nation psychological study, realizado por James Georgas, John Berry, Van de Vijver, Çigdem Kagitçibasi e Ype Poortinga, publicado em 2006. O referente estudo comparou dados oriundos de 30 países sobre o modo de vida familiar a partir de duas questões básicas: 1. quais as semelhanças e diferenças entre as famílias localizadas nas várias partes do mundo? Haveriam universais?; 2. estaria havendo uma mudança do padrão de vida familiar no que denominam Mundo Majoritário para um padrão único Ocidental ${ }^{1}$

A obra em lide consiste em capítulos escritos por diferentes combinações dos autores, os quais descrevem as sucessivas etapas do estudo. Na segunda parte do livro, por eles denominada de estudo qualitativo, são apresentados os dados referentes a 30 países, o Brasil sendo um deles (TORRES; DESSEN, 2006).

Segundo Georgas, Berry, Vijver, Kagitçibasi e Poortinga (2006), inúmeros estudos de antropólogos e sociólogos, nos últimos 200 anos, apontaram para mudanças mundiais na estrutura familiar. Seu maior determinante foi a crescente afluência nas sociedades, primariamente como resultado da industrialização e da urbanização. Criticam a definição estrutural de família baseada na demografia como não apreendendo que esta instituição vive em redes, mesmo que habitando em moradias isoladas. Raramente tais estudos investigam relações de parentesco. A presença de redes familiares pode significar que essas alterações não indicariam, necessariamente, uma mudança nos sistemas familiares. Entre nós, vários autores (CARVALHO; BASTOS; RABINOVICH; SAMPAIO, 2006; SARTI, 2004; FONSECA, 2004; RABINOVICH, BASTOS, 2007), têm enfatizado igualmente o conceito de rede sócio-familiar como correspondendo à família no Brasil.

A teoria da modernização, e mais recentemente, a da globalização, previu a convergência de sistemas familiares extensos a nucleares, como na Europa e nos Estados Unidos. No entanto, Inkeles (1998, apud GEORGAS et al., 2006), um dos proponentes da teoria da modernização, questionou o grau em que o desenvolvimento econômico crescente dos países leva à convergência em todos os domínios das instituições sociais. Haveria forças culturais que resistem à convergência, a família sendo uma destas instituições sociais devido ao fato de nela persistirem valores de alta ordem. Fornece, como exemplo desta resistência à mudança, o achado da pesquisa de que os maridos não ajudam as esposas em tarefas domésticas, mesmo em sociedades industriais avançadas e após as esposas terem entrado no mercado de trabalho.

Apoiados nos pensamentos de Berry e Poortinga (2006), o estudo adotou o conceito de modelo ecocultural. Tal modelo propõe levar em 
conta a diversidade psicológica humana, considerando as diferenças e similaridades entre pessoas e entre grupos. Para tanto, utiliza duas fontes fundamentais de influências - ecológica e sócio-política -, e dois aspectos de populações humanas adaptados a elas: características culturais e biológicas. Essas variáveis da população são passadas aos indivíduos por diversas "variáveis de transmissão", tais como enculturação, socialização, genética e aculturação. Berry e Poortinga (2006) consideram a cultura como adaptação evolutiva a influências ecológicas e sócio-políticas e percebem as características psicológicas individuais em uma população como adaptativas a seu contexto cultural.

Como uma instituição cultural, a família pode ser vista como adaptativa ao contexto ecocultural e como um veículo para a transmissão cultural. A família, assim, ocupa um lugar central na abordagem ecocultural, ligando contextos básicos ao desenvolvimetno comportamental do indivíduo.

Kagitçibasi (2006) propõe o modelo de interdependência psicológica, que integra a necessidade de autonomia, predominante no mundo ocidental, e a de relacionamento, predominante no Mundo Majoritário. Para ela, sociedades mantendo valores individualistas, e refletindo-os em seus padrões familiares e de criação de crianças, reconheceram e reforçaram a necessidade básica humana por autonomia, ignorando em alguma medida a necessidade humana igualmente básica por intimidade/conexão. Sociedades que reforçaram valores coletivistas fizeram o contrário. Segundo a autora, reconhecer ambas as necessidades humanas promete contribuir para um melhor bem-estar das pessoas.

Portanto, Georgas et al. (2006) questionam a globalização como resultando inevitavelmente na convergência de tipos familiares, perguntando-se se os fatores culturais e necessidades psicológicas relacionadas a vínculos emocionais com parentes seriam suficientemente fortes para manter a diversidade nos tipos de família, a despeito da convergência nos sistemas econômicos e sociais dos países.

Para responder a tais questões, os autores propuseram estudar as relações entre variáveis culturais, papéis familiares e variáveis psicológicas. Para tanto, utilizaram uma série de instrumentos de pesquisa: (a) um questionário estruturado sobre papéis, comportamentos, atividades e relações entre os membros da família, enfocando pai, mãe, avós, tio/tia, menino e menina de 10 anos, moça e rapaz de 20 anos; (b) Cinco Fatores de Traços de Personalidade: roteiro que aborda os traços extroversão, agradabilidade, consciência, estabilidade emocional e abertura à experiência; (c) Valores: roteiro sobre os valores inserção, conservadorismo, harmonia, compromisso igualitário, autonomia intelectual, autonomia afetiva e domínio; (d) Escala de auto-construção: abordando o self 
independente e interdependente; (e) Valores familiares: sobre papéis hierárquicos de pai e mãe, além das relações com família e parentes; (f) Distância emocional do participante com seus familiares.

Entre as inúmeras conclusões parciais obtidas pelos autores, no que se refere a uma combinação de diferenças e similaridades entre as famílias, apontamos primeiramente essas diferenças relacionadas especialmente ao aspecto sócio-econômico dos países; depois, as semelhanças relativas aos vínculos emocionais, presentes em todas as famílias, embora com variações.

Segundo Arriagada (2000), uma das modificações mais importantes nas famílias latino-americanas, nas últimas décadas, foi o declínio do modelo patriarcal de família, caracterizado pela autoridade exercida pelo homem sobre a mulher e os filhos, associado principalmente à entrada maciça das mulheres no mercado de trabalho. A decadência do sistema patriarcal está ligada também ao fim do sistema de única contribuição financeira no lar, pois mulheres, jovens e, por vezes, até as crianças foram inseridos no mercado de trabalho. Além disso, também aumenta o número de lares chefiados por mulheres que, em sua maioria, são monoparentais.

As famílias brasileiras reagem ou adaptam-se a tais mudanças (CERVENY, 2007; DESSEN, POLÔNIA, 2007). Torres e Dessen (2006) afirmam que as transformações que ocorreram nas famílias brasileiras resultaram não apenas em mudanças estruturais, mas, também, na redefinição do modelo tradicional de família nuclear. Na medida em que tais transformações estão ainda ocorrendo, não é possível definir com clareza um novo modelo de funcionamento para as famílias brasileiras, em razão das mudanças na sociedade de modo geral.

No entanto, ao pretender comparar 30 países, Georgas et al. nomes exponenciais na psicologia inter-cultural e no estudo da família - (2006) necessitaram igualar as diferenças, ou seja, a partir de uma uniformização quanto aos objetivos e métodos utilizados. Com isto, potenciais diversidades não puderam aparecer. Assim, entendemos ser pertinente aprofundar a discussão por eles empreendida por meio de metodologia qualitativa - questões abertas - aplicada em um contexto societário nordestino brasileiro. O método qualitativo pressupõe, em princípio, o acesso ao que não se sabe.

Portanto, o objetivo do presente estudo foi cotejar os achados do estudo de Georgas et al. (2006) pela introdução de questões abertas quanto à composição, definição e ideal de família dos participantes. Pretendemos, assim, compreender aspectos que estariam inerentes às respostas obtidas por meio da metodologia quantitativa. 


\section{Metodologia}

\subsection{Local e participantes}

Participaram desta pesquisa 170 universitários do Estado da Bahia (Brasil), sendo: 91 da capital $(53,5 \%)$ e $79(46,5 \%)$ do interior do Estado; sendo 122 oriundos de Instituições de Ensino Superior (IES) particulares e 48 de públicas; 127 do sexo feminino e 43 do masculino e as idades variaram de 17 a 62 anos, sendo a média de 25,9 anos.

Os estudantes estavam matriculados em cursos de diferentes áreas: Pedagogia (trinta e sete participantes), Enfermagem (trinta), Engenharia de Produção (vinte e sete), Serviço Social (dezoito), Licenciatura em Matemática (dezessete), Psicologia (catorze), Direito (onze), Administração (quatro), Medicina (quatro) Letras (dois), Análise de Sistemas (um) e cinco universitários não informaram o curso.

Em Salvador, dados foram coletados em cinco IES, todas particulares e, no interior, a coleta se deu em uma IES particular, localizada no município de Alagoinhas, e em duas públicas, uma em Feira de Santana e outra em Ilhéus. Salvador é a terceira metrópole brasileira, com 2.998.056 habitantes, Feira de Santana tem 591.707, Ilhéus tem 219.266 e, finalmente, Alagoinhas tem 137.810 habitantes. Em todas as cidades, a economia está mais voltada para a área de serviços.

$\mathrm{O}$ instrumento elaborado consistiu nas seguintes questões abertas: 0 que é uma família para você?; Quem faz parte de sua família?; O que é uma família ideal para você?

A coleta de dados foi realizada por alunos ${ }^{2}$ regulares e especiais do Programa de Pós-Graduação em Família na Sociedade Contemporânea que cursaram a disciplina Contextos Familiares: vínculos de identidade e de pertencimento, no segundo semestre de 2009, ministrada pelas autoras do presente artigo.

Os participantes foram contatados nas Instituições de Ensino Superior em que estudavam. Após ser explicado o objetivo da pesquisa, os questionários foram respondidos em sala de aula, na presença de um dos pesquisadores.

Os participantes assinaram Termo de Consentimento Livre e Esclarecido, sendo resguardado o anonimato dos respondentes e preservada a dignidade humana deles. O projeto de pesquisa foi submetido e aprovado pelo Comitê de Ética em Pesquisa da Universidade Católica do Salvador.

O tratamento das questões abertas foi realizado através da análise de conteúdo. Elaborou-se categorias com base na identificação dos termos aglutinadores contidos nas respostas. Tais termos foram reunidos segundo os seus significados. 


\section{Definindo a família: quem faz parte de sua família}

Referente às respostas às questões abertas, um resultado instigante foi a categorização à questão: quem faz parte de sua família (Tabela 1). Dos 170 participantes, dois não responderam.

Tabela 1. Distribuição porcentual das respostas dos participantes à questão "quem faz parte da sua família?". Bahia, 2009.

\begin{tabular}{l|c}
\hline \multicolumn{1}{c|}{ CATEGORIAS } & Frequência/Porcentagens \\
\hline Extensa & $76(45,5 \%)$ \\
Nuclear & $39(23,3 \%)$ \\
Extensa + amigos & $18(10,7 \%)$ \\
Ampliada & $11(06,5 \%)$ \\
Monoparental ampliada & $10(05,9 \%)$ \\
Monoparental & $07(04,1 \%)$ \\
Ligações afetivas & $03(01,7 \%)$ \\
Tia & $02(01,1 \%)$ \\
Reconstituída & $01(0,6 \%)$ \\
Reconstituída extensa & $01(0,6 \%)$ \\
\hline TOTAL & $168(100 \%)$ \\
\hline
\end{tabular}

Obtivemos 10 tipos de família, em que o agrupamento extenso correspondeu a $45,5 \%$, e a nuclear a apenas $23,3 \%$. Os modos de vida familiar extenso atingem o total de $63,3 \%$ se considerarmos as categorias extensa + amigos, ampliada e reconstituída extensa. Estas respostas indicam, claramente, a riqueza das combinações do que se denomina família.

O estudo original enfatizou a importância de muitos estudos demográficos não se referirem a relações de parentesco ou a redes familiares, temática que temos igualmente apresentado como característica do modo de viver no Brasil (RABINOVICH, BASTOS, 2007). Para Georgas (2006, p. 235), dados demográficos têm confundido membros de família nuclear e extensa, diminuindo a importância das redes familiares. Afirmam que "estas são algumas das razões porque a metodologia do presente estudo não incluiu definições estruturais de família", baseadas em dados demográficos sobre tipos de famílias. Empregam, outrossim, o conceito de Murdock de família nuclear como um núcleo dentro da constelação de famílias nucleares que formam uma família extensa. Isto é, a família nuclear é uma entre núcleos de uma constelação de dois núcleos paralelos 
dos avós de cada lado da família, junto com os tios e tias, irmãs e irmãos, primos, etc. Assim, o estudo referência focalizou a distância entre os membros da família e a quantidade de comunicação por telefone e visitas.

Nossos resultados igualmente levam a um questionamento do que se denomina estrutura familiar, reforçando o que os autores do estudobase apontam como crítica à visão demográfica de definição de família.

\section{Definindo a família: o que é família para você}

Na Tabela 2, a seguir, temos as respostas do que os respondentes definem como família. Dos 170 participantes, dois não responderam.

Tabela 2. Distribuição porcentual das respostas dos participantes à questão "o que é família para você?". Bahia, 2009.*

\begin{tabular}{l|c}
\hline \multicolumn{1}{c|}{ CATEGORIAS } & Frequência/Porcentagens \\
\hline Base segura & $137(59,1 \%)$ \\
Definição de família como rede social & $29(12,5 \%)$ \\
Definição de família tradicional & $18(07,8 \%)$ \\
Compartilha valores/ideais & $18(07,8 \%)$ \\
Formação da cidadania & $15(06,5 \%)$ \\
Definição de família como parentesco & $08(03,4 \%)$ \\
Base econômica & $04(01,7 \%)$ \\
Aspectos negativos & $03(01,2 \%)$ \\
\hline TOTAL & $232(100 \%)$ \\
\hline
\end{tabular}

* Pode incluir mais de uma resposta por participante.

Agregamos vários conteúdos fornecidos em uma categoria geral denominada "base segura", apoiando-nos no conceito de Bowlby (1984). De acordo com esse autor, o comportamento de apego é um processo ativo de adaptação baseado em estratégias de satisfação das necessidades de segurança e proteção conquistadas através de uma proximidade segura com um adulto, capacitado a proteger a criança de perigos iminentes do ambiente. Este processo de apego aumenta a capacidade infantil para elaborar pensamentos e organizar comportamentos quando a criança observa modelos e interações que, mais tarde, introduzem-na no processo de socialização, quando demonstrará compartilhamento e cooperação com os demais. Este conjunto de interações adulto/criança que a capacitam para enfrentar a separação das pessoas de quem depende passou a ser denominada base segura. Foram reunidos sob esta denominação os seguintes descritores que qualificam esse conceito: base da pessoa, afeto, tudo, suporte incondicional, sustento, vínculo, cuidado e proteção e felicidade/tudo de bom, perfazendo o total de $59,1 \%$ das respostas. 
Na sequência, com uma porcentagem bem menor (12,5\%), obtevese a definição de família como rede social que agrupa os descritores convivência e afinidade.

A família tradicional (que agrupa respostas que a definem como reunindo pessoas do mesmo sangue e mesma tradição, mais baseada na biologia) foi encontrada em $7,8 \%$ das respostas. A mesma porcentagem foi obtida em instituição que compartilha valores/ideais. Os aspectos negativos (dependência e alienação para a pessoa, instituição se deteriorando e empresa) apareceram em apenas 1,2\% das respostas. Isso sinaliza que, embora a instituição familiar seja alvo de muitas críticas, ela é tida pela maioria esmagadora das respostas como positiva.

\section{Definindo família: o que é uma família ideal para você}

O interesse maior da tabela 2 ocorre quando comparada com as respostas à questão sobre o que seria uma família ideal para os nossos respondentes (Tabela 3). Dos 170 participantes, dois não responderam a questão e nove afirmaram que "não existe família ideal".

Tabela 3. Distribuição porcentual das respostas dos participantes à questão "o que é uma família ideal para você?". Bahia, 2009.*

\begin{tabular}{|c|c|}
\hline CATEGORIAS & Frequência/Porcentagens \\
\hline Convivência harmoniosa & $162(52,3 \%)$ \\
\hline Afeto & $55(17,7 \%)$ \\
\hline Suporte incondicional & $37(11,9 \%)$ \\
\hline Apoio & $20(06,5 \%)$ \\
\hline Cuidado/proteção & $13(04,2 \%)$ \\
\hline Valores, religiosidade & $06(01,9 \%)$ \\
\hline Humor & $05(01,6 \%)$ \\
\hline Sustento & $03(01,0 \%)$ \\
\hline Composição nuclear & $03(01,0 \%)$ \\
\hline Crescimento/desenvolvimento & $03(01,0 \%)$ \\
\hline Composição extensa & $01(0,3 \%)$ \\
\hline $\begin{array}{l}\text { Outros (independência e } \\
\text { estabelece limites) }\end{array}$ & $02(0,6 \%)$ \\
\hline TOTAL & $310(100 \%)$ \\
\hline
\end{tabular}


diálogo e cooperação. Elementos como afeto (17,7\%), suporte incondicional $(11,9 \%)$, apoio $(6,5 \%)$ e cuidado/proteção $(4,2 \%)$ sustento $(0,9)$ perfazem $40,3 \%$ das respostas e podem ser agrupados como base segura. Ou seja: há uma mudança de padrão quanto ao significado de família que adquire uma conotação de horizontalidade em contraposição a uma de verticalidade (embora ainda existente), ou seja, de um padrão de organização hierárquico para um democrático (Tabela 4).

Tabela 4. Comparação entre categorias agrupadas em definição de família e definição ideal de família, Bahia, 2009.

\begin{tabular}{l|cc}
\hline CATEGORIAS & \% Definição de família & $\begin{array}{c}\text { \% Definição de família } \\
\text { ideal }\end{array}$ \\
\hline Base segura & 59,1 & 40,3 \\
Convivência harmoniosa & $20,3 *$ & 52,3 \\
\hline * Agrupou definição de família como rede social & $(12,5 \%)$ & e compartilha \\
valores/ideais (7,8\%) da Tabela 4. &
\end{tabular}

Georgas et al (2006) formulam a hipótese de que a mudança na família ocorre devido à mudança nos valores familiares do papel hierárquico de mãe e pai. Poortinga e Georgas (2006), a respeito da autoridade e poder na família, apontam que uma importante característica que sobressai nos retratos tradicionais autocráticos é o poder do pai na família. Exemplificam tal característica por meio do relato de Torres e Dessen (2006) sobre a família no Brasil, em que estes são explícitos em atribuir ao marido e pai o poder que ele exerce sobre sua esposa e filhos. Embora fortemente relacionados ao nível sócio-econômico dos países, em certos países de nível médio que, até recentemente, eram culturas agrícolas, há uma forte rejeição de valores de papel hierárquicos relacionados principalmente ao poder autocrático do pai nas famílias extensas agrícolas.

Sugerem esses autores que valores relativos às relações hierárquicas na família, e valores hierárquicos em geral, são os mais importantes indicadores de mudança na família, conforme pudemos confirmar em nosso estudo qualitativo sobre o significado de família. Para esses autores, os respondentes jovens parecem rejeitar o modelo autoritário do pai que toma todas as decisões na família e o papel da mãe e filhos submissos e acomodados, uma tendência também relatada nos retratos dos países. Apontam ser significativo que este padrão prevaleceu nos países de nível médio de afluência, como o Brasil, sugerindo mudanças em aspectos funcionais da família. Por outro lado, valores familiares relacionados à manutenção de relações com os membros familiares e parentes mudaram muito menos do que os papéis hierárquicos na família nuclear. A autonomia que 
emerge nas famílias em mudança aparecem em setores mais desenvolvidos, urbanos e educados, de países menos afluentes. Contudo, o Modelo de Mudança na Família de Kagitçibasi (2006) propõe que a autonomia emerge (e uma menor aceitação da hierarquia), mas o relacionamento emocional e afetivo continua a ser importante no Modelo de Família de Interdependência Emocional.

Nenhum dos 30 relatos menciona mudanças na direção do fortalecimento da autoridade do pai (ou do sexo masculino); todas as tendências são mais para a partilha do poder entre pai e mãe, embora isso certamente não signifique que a igualdade foi atingida. A razão mais comum para as alterações mencionadas explicitamente por um quarto dos autores é o emprego de mulheres fora de casa e o aumento do seu nível de educação, permitindo-lhes fazer uma contribuição econômica para a família. Em dois terços dos retratos são feitas referências a um duplo papel da mulher na família contemporânea, relacionado ao aumento da educação das mulheres e da sua importância crescente na força de trabalho. Esta situação enfraqueceu o papel tradicional do pai; a distribuição de papéis tradicionais com o pai como provedor e a mãe na casa com as crianças é descrita como válida hoje em apenas retratos de quatro países.

Para Arriagada (2000), novas relações entre pais e filhos conduzem a um aumento dos direitos dos filhos e perda da relevância das relações de hierarquia e de submissão, aparecendo o direito individual e a ênfase na relação pessoal acima dos interesses familiares, com lentas mudanças de gênero, tanto na distribuição do poder e nas políticas públicas quanto no interior da família.

Segundo Torres e Dessen (2006), Schwartz, aplicando a sua escala de valores, observou que brasileiros tiveram resultado baixo em autonomia intelectual e emocional, correspondendo à dimensão do individualismo e alto em conservadorismo e hierarquia. Estes autores apontam que outros estudos encontraram que os brasileiros eram significativamente mais coletivistas do que outros países, havendo pouca participação em tomadas de decisão e preferência pelo verticalismo. Portanto, a cultura nacional seria vertical-coletivista. Neste padrão cultural, a desigualdade não seria apenas aceita como esperada. Os autores sugeriram uma variação neste padrão cultural que se tornaria crescentemente individualista e menos vertical na medida em que se vai do norte ao sul do país.

No entanto, em artigo recente, Dessen (2010) realiza uma re-leitura de estudos anteriores sobre o papel do pai no Brasil, perguntando se as concepções de pai e mãe ideais "dos jovens das décadas de 70 e 80 se transformaram no pai real e na mãe real dos dias atuais" ( $p$. 210). Considera que grandes mudanças ocorreram no papel das mulheres, afetando significativamente os papéis masculinos, levando a uma hierarquia mais flexível em direção de uma maior igualdade 
nas relações conjugais e parentais. Além disso, esta autora advoga que o sistema complexo que é a família "sofre variações, em razão dos contextos cultural, social e histórico em que está inserida" ( $p$. 210). Assim, as afirmações sobre as variações no território, bem como a cultura nacional como um processo vertical-coletivista, estão sendo re-examinadas.

Um destes contextos é certamente o macro-econômico e suas relações com o universo do trabalho, o principal responsável pelas mudanças femininas e de suas consequências na vida familiar. Assim, Souza e Lamounier (2010), estudando a "nova" classe média brasileira, apontam que esta assume posturas independentes como desejar ter o próprio negócio e a própria poupança, advogando ao mesmo tempo fortemente a ação estatal. $O$ resultado mais importante deste estudo foi que tal classe média não copia a classe chamada A, mas tem padrões e gostos próprios.

\section{Conclusão}

Os jovens baianos, ao indicarem como ideal de família uma família mais igualitária, em que a convivência harmoniosa a define, aparece como um indicador de um movimento no ethos familiar e social, mesmo que ainda em nível de desejo. No entanto, como sugerido por Dessen (2010), o desejo pressupõe, em certa medida, a sua realização, donde podemos supor que existe na sociedade baiana uma força potencial de mudança em direção a um sistema igualitário de relacionamentos, em que há o desejo de relações compartilhadas, além de uma compreensão amplificada do que seja o vínculo afetivo ou, pelo menos, de qual seja a sua base, decorrente dos inúmeros arranjos familiares relatados pelos respondentes.

A importância deste estudo, que pode ser uma pesquisa piloto, é abrir uma série de questionamentos, dos quais poderemos elencar alguns: como seria a definição de família ideal e real em outros segmentos sócio-educativos, na Bahia e em outros estados? Que diferenças poderiam ser encontradas nos quesitos pequisados nos diversos estados brasileiros? Será que homens e mulheres divergem quanto a esta concepção? Variará ela com a idade do respondente? Como a definição de família é influenciada pela história da família, por exemplo, ser oriunda de imigração ou de migração? Como as diversas etnias, no sentido da diversidade cultural, manifestar-se-ia nas resposta ao que é família, real e ideal? Como a proximidade ou a distância entre membros familiares influenciaria na definição da família? Como definir melhor os termos empregados pelos entrevistados? Sabemos já que nas cidades do Rio de Janeiro e São Paulo as relações intra-familiares, em sua maioria, estão se dando de modo democrático: o que estará ocorrendo nas demais cidades, quer 
do interior, quer do Norte, do Nordeste, do Sul, do Centro-Oeste? O que estaria subentendido de tais diferenças em relação aos contextos sócio-culturais e históricos em que elas ocorrem?

\section{Referências}

ARRIAGADA, I. Nuevas familias para un nuevo siglo? Cadernos de Psicologia e Educação: Paidéia, Ribeirão Preto, v. 18, n. 10, p. $28-$ 35, 2000.

BERRY, J. W.; POORTINGA, Y. H. Cross-cultural theory and methodology. In: GEORGAS, J.; BERRY, J. W.; VIJ NER, F. J. R. V. D.; KAGIÇIBASI Ç.; POORTINGA Y. H. Families across culture. A 30nation psychological study. Cambridge: Cambridge University Press, 2006, p. 51-71.

BOWLBY, J. Apego. São Paulo: Martins Fontes, 1984.

CARVALHO, A. M. A.; BASTOS, A. C. S.; RABINOVICH, E. P.;

SAMPAIO, S. M. R. Vínculos e redes sociais em contextos familiares e institucionais: uma reflexão conceitual. Psicologia em Estudo, Maringá, v. 11, n. 3, p. 589-598, setembro-dezembro, 2006.

CERVENY, C. M. d. O. Apresentação - palavras introdutórias. In: 2007, p. 09-16.

Família em movimento. São Paulo: Casa do Psicólogo,

DESSEN, M. A. Estudando a família em desenvolvimento: desafios conceituais e teóricos. Psicologia, Ciência e Profissão, Brasília, v. 30, 2010, p. 202-219.

DESSEN, M. A; Polônia, A. C. A família e a escola como contextos de desenvolvimento humano. Paidéia, Ribeirão Preto, v. 17, n. 36, p. 21-32, 2007.

FONSECA, C. (2004). Fabricando família: políticas públicas para o acolhimento de jovens em situação de risco. In: JACQUET C.; COSTA L. F. (Org.), Família em mudança. São Paulo: Companhia Ilimitada, 2004, p. 215-244.

GEORGAS, J.; BERRY, J. W.; VIJ VER, F. J. R. V. D.; KAGITÇIBASI, Ç.; POORTINGA, Y. H. (2006). Families across cultures. A 30-nation psychological study. Cambridge: Cambridge University Press.

KAGITÇIBASI, Ç. Theoretical perspectives on family change. In: GEORGAS, J.; BERRY, J. W.; VIJ NER, F. J. R. V. D.; KAGIÇIBASI, Ç.; POORTINGA, $Y$. H., Families across cultures. A 30-nation psychological study. Cambridge: Cambridge University Press, 2006, p. 72-89.

POORTINGA, Y. H.; GEORGAS, J. Family portraits from 30 countries: an overview. In: GEORGAS, J.; BERRY, J. W.; VIJ NER, F. J. R. V. D.; KAGIÇIBASI, Ç.; POORTINGA, Y. H. Families across culture. A 30nation psychological study. Cambridge: Cambridge University Press, 2006, p. 90-99. 
RABINOVICH, E. P.; BASTOS, A. C. S. Famílias e projetos sociais: analisando essa relação no caso de um quilombo em São Paulo. Psicologia em Estudo, Maringá, v. 12, n. 1, p. 3-11, 2007.

SARTI, C. A. Algumas questões sobre família e políticas sociais. In: JACQUET, C.; COSTA, L. F. (Org.), Família em mudança São Paulo: Companhia Ilimitada, 2004, p. 193-214.

SOUZA, A. D., LAMOUNIER, B. A classe média brasileira. Ambições, valores e porjetos de sociedade. Rio de Janeiro: Ed. Campus, 2010.

TORRES, C. V.; DESSEN, M. A. The Brazilian jeitinho: Brazil's subcultures, its diversity of social contexts, an its family structures. In: GEORGAS, J.; BERRY, J. W.; VIJ NER, F. J. R. V. D.; KAGIÇIBASI, Ç.; POORTINGA, $Y$. H. Families across culture. A 30-nation psychological study. Cambridge: Cambridge University Press, 2006, p. 259-266.

\section{Endereço para correspondência Elaine Pedreira Rabinovich}

Programa de Pós-graduação em Família na Sociedade Contemporânea (UCSal) Av. Cardeal da Silva n. 205 - Federação - CEP: 40.231-902 - Salvador - Ba. Endereço eletrônico: elainepr@brasmail.com.br

\section{Anamélia Lins e Silva Franco}

Programa de Pós-graduação em Família na Sociedade Contemporânea (UCSal) Av. Cardeal da Silva n. 205 - Federação - CEP: 40.231-902 - Salvador - Ba. Endereço eletrônico: anameliafranco@uol.com.br

\section{Lúcia Vaz de Campos Moreira}

Programa de Pós-graduação em Família na Sociedade Contemporânea (UCSal) Av. Cardeal da Silva n. 205 - Federação - CEP: 40.231-902 - Salvador - Ba. Endereço eletrônico: luciavcm@oi.com.br

Recebido em: 16/03/2010

Reformulado em: 16/02/2011

Aceito para publicação em: 11/04/2011

Acompanhamento do processo editorial: Ariane Patrícia Ewald

\section{Notas}

* Doutora em Psicologia (USP) e professora do Programa de Pós-graduação em Família na Sociedade Contemporânea da Universidade Católica do Salvador. **Doutora em Saúde Pública pelo Instituto de Saúde Coletiva (UFBa) e professora do Programa de Pós-graduação em Família na Sociedade Contemporânea da Universidade Católica do Salvador.

*** Doutora em Psicologia (USP) e professora do Programa de Pós-graduação em Família na Sociedade Contemporânea da Universidade Católica do Salvador. ${ }^{1}$ Conforme Kagitçibasi (2006), o termo ocidental está sendo usado por faltar outro melhor para se referir a populações preponderantemente de classe média em contextos ecoculturais da América do Norte e da Europa Ocidental, com um pleno reconhecimento de que há uma ampla diversidade no "Ocidente". Similarmente, "não-ocidental" ou "Mundo Majoritário" é utilizado para se referir ao restante da população mundial, que também apresenta ampla variação. 
Elaine Pedreira Rabinovich, Anamélia Lins e Silva Franco, Lúcia Vaz de Campos Moreira Compreensão do significado de família por estudantes universitários baianos

${ }^{2}$ As autoras agradecem as alunas que colaboraram na coleta e discussão de dados: Ana Barreiros de Carvalho, Erica Santana, Helaine de Souza, Jamaica Santana, Joana Torres, Juracyara Santana, Kátia Almeida, Maria Constança Cajado, Maria Elisa Medeiros, Maria Goretti Cruz, Mariana Rosa, Marilaine Ferreira, Moacir Oliveira, Noemi Fontes, Teresa Leal e Vânia Almeida. 